\title{
Rotating system for four-dimensional transverse rms-emittance measurements
}

\author{
C. Xiao, M. Maier, X. N. Du, P. Gerhard, L. Groening, S. Mickat, and H. Vormann \\ GSI Helmholtzzentrum für Schwerionenforschung GmbH, D-64291 Darmstadt, Germany
}

(Received 1 February 2016; published 19 July 2016)

\begin{abstract}
Knowledge of the transverse four-dimensional beam rms parameters is essential for applications that involve lattice elements that couple the two transverse degrees of freedom (planes). Of special interest is the elimination of interplane correlations to reduce the projected emittances. A dedicated rotating system for emittance measurements (ROSE) has been proposed, developed, and successfully commissioned to fully determine the four-dimensional beam matrix. This device has been used at the high charge injector (HLI) at GSI in a beam line which is composed of a skew quadrupole triplet, a normal quadrupole doublet, and ROSE. Mathematical algorithms, measurements, and the analysis of errors and the decoupling capability for ion beams of ${ }^{83} \mathrm{Kr}^{13+}$ at $1.4 \mathrm{MeV} / \mathrm{u}$ are reported in this paper.
\end{abstract}

DOI: 10.1103/PhysRevAccelBeams.19.072802

\section{INTRODUCTION}

Emittance is an important figure of merit for propagation of charged particle beams. It is defined as the amount of phase space being occupied by the particle distribution to quantify the beam quality and to match the following optics. Precise knowledge from measurements of particle distribution parameters is important for accelerator design and for phase-space manipulation. However, most of the published work is just on separated measurements of twodimensional $x-x^{\prime}$ and $y-y^{\prime}$ subphase spaces (planes) [1-3]. For simplicity, correlations between the two planes, i.e. $x-y, x-y^{\prime}, x^{\prime}-y$, and $x^{\prime}-y^{\prime}$ are often assumed as zero. However, such interplane correlations may be produced by interplane coupling fields such as dipole fringes, solenoids, and titled magnets [4] or just by beam losses.

Ion beams extracted from electron cyclotron resonance (ECR) ion sources have a complex structure in the fourdimensional phase space [5]. Distributions with equal projected rms emittances are strongly correlated after extraction [6-8]. Correlations increase the projected rms emittances. Removing correlations reduces the effective emittances without introduction of beam loss through scraping for instance. In order to remove unknown correlations, they must be quantified by measurements. Accordingly, four-dimensional diagnostics has the major task of allowing for elimination of interplane coupling.

For beam lines comprising just noncoupling elements as upright quadrupoles, dipoles, and accelerating gaps, the horizontal and vertical beam dynamics are decoupled. The design of such beam lines can be accomplished ignoring

Published by the American Physical Society under the terms of the Creative Commons Attribution 3.0 License. Further distribution of this work must maintain attribution to the author(s) and the published article's title, journal citation, and DOI. eventual interplane correlations in the beam as long as the projected distribution parameters are known at the beam line entrance. This convenience of ignoring correlations cannot be further afforded, if the beam line includes coupling elements as solenoids or skew quadrupoles for instance. In those cases even the projected distribution parameters along the beam line will depend on interplane correlations at the entrance of the beam line [9].

Using standard slit and grid emittance measurement devices [10,11] and multislit or screen devices [12] such as the pepper-pot technique, the projected phase-space distributions, i.e. the horizontal and vertical rms emittances, can be measured. The slit determines the location of the phase-space element. A subsequent grid measures the angular distribution of the ions that passed the slit. By moving the slit and recording the angular distribution at each slit position, the projected phases-space distribution is measured. The direction of movements of the slit and grid determines the plane onto which the four-dimensional distribution is projected. Interplane correlation matrix elements cannot be measured directly using a slit and grid configuration.

There is considerable work on measuring fourdimensional distributions using pepper-pots [13-15] for ion beams at energies below $150 \mathrm{keV} / \mathrm{u}$, where the beam is stopped by the pepper-pot mask. However, this method is not applicable at energies beyond $150 \mathrm{keV} / \mathrm{u}$ due to technical reasons, i.e. doubtful readout by temperature-dependent screens and fixed resolutions by holes and screens [16]. Four-dimensional emittance measurements were proposed and conducted for instance in [17-21] at electron machines. Other options based on a phase space tomography technique have been developed to reconstruct the two-dimensional phase space in [22] and the full four-dimensional phase space in $[23,24]$. The combination of skew quadrupoles with a slit and grid emittance measurement device has been applied 
successfully for high intensity uranium ions at an energy of $11.4 \mathrm{MeV} / \mathrm{u}$ at GSI [25]. This paper reports on a method without skew quadrupoles that features reduced time (about a factor of 3) needed to perform the measurements within about one hour.

The paper starts with an introduction of the parameters that quantify four-dimensional particle distributions. Section III is on the method of a rotating system for emittance measurements (ROSE); analytical calculation and numerical analysis are elaborated comprehensively. Section IV is on the commissioning method and software for measuring and evaluating the full four-dimensional beam matrix. Section V shows the capability of ROSE to provide the input for successful elimination of interplane coupling.

\section{FOUR-DIMENSIONAL RMS QUANTITIES}

Four-dimensional beam rms-emittance measurements require determination of ten unique elements of the second moments beam matrix. The $4 \times 4$ symmetric second moments beam matrix $C$ can be expressed as [26]

$$
C=\left[\begin{array}{cccc}
\langle x x\rangle & \left\langle x x^{\prime}\right\rangle & \langle x y\rangle & \left\langle x y^{\prime}\right\rangle \\
\left\langle x^{\prime} x\right\rangle & \left\langle x^{\prime} x^{\prime}\right\rangle & \left\langle x^{\prime} y\right\rangle & \left\langle x^{\prime} y^{\prime}\right\rangle \\
\langle y x\rangle & \left\langle y x^{\prime}\right\rangle & \langle y y\rangle & \left\langle y y^{\prime}\right\rangle \\
\left\langle y^{\prime} x\right\rangle & \left\langle y^{\prime} x^{\prime}\right\rangle & \left\langle y^{\prime} y\right\rangle & \left\langle y^{\prime} y^{\prime}\right\rangle
\end{array}\right],
$$

where $x$ and $y$ are the horizontal and vertical coordinates, respectively, and $x^{\prime}$ and $y^{\prime}$ are their derivatives with respect to the longitudinal coordinate.

Four of the matrix elements quantify the coupling. If at least one of the elements of the off-diagonal submatrix is nonzero, the beam is transversely coupled. Projected rms emittances $\varepsilon_{x}$ and $\varepsilon_{y}$ are quantities which are used to characterize the transverse beam quality in the laboratory coordinate system and are invariant under linear uncoupled (with respect to the laboratory coordinate system) symplectic transformations. Projected rms emittances are the rms phase space areas from projections of the particle distribution onto the planes, and their values are equal to the square roots of the determinants of the on-diagonal $2 \times 2$ submatrices, i.e. phase space area divided by $\pi$ :

$$
\varepsilon_{\mu}=\sqrt{\langle\mu \mu\rangle\left\langle\mu^{\prime} \mu^{\prime}\right\rangle-\left\langle\mu \mu^{\prime}\right\rangle^{2}},
$$

where $\mu$ refers to either $x$ or $y$. The dimensionless parameter $\alpha$ relates to the $\mu-\mu^{\prime}$ correlation and the $\beta$ function refers to the beam width. They are defined as

$$
\alpha_{\mu}=-\frac{\left\langle\mu \mu^{\prime}\right\rangle}{\varepsilon_{\mu}}, \quad \beta_{\mu}=\frac{\langle\mu \mu\rangle}{\varepsilon_{\mu}} .
$$

The eigen emittances $\varepsilon_{1}$ and $\varepsilon_{2}$ are invariant under coupled linear symplectic transformations provided by solenoids or skew quadrupoles for instance [27]. None of the projected emittances can be smaller than the smaller of the two eigen emittances. The eigen emittances can be expressed as [28]

$$
\varepsilon_{1,2}=\frac{1}{2} \sqrt{-\operatorname{tr}\left[(C J)^{2}\right] \pm \sqrt{\operatorname{tr}^{2}\left[(C J)^{2}\right]-16|C|}}
$$

The square matrix $J$ is the skew-symmetric matrix with nonzero entries in the block diagonal off form and $J$ is defined as

$$
J:=\left[\begin{array}{cccc}
0 & 1 & 0 & 0 \\
-1 & 0 & 0 & 0 \\
0 & 0 & 0 & 1 \\
0 & 0 & -1 & 0
\end{array}\right]
$$

The eigen emittances are equal to projected rms emittances if and only if all interplane correlations are zero. If the second moments beam matrix has correlations between horizontal and vertical phase spaces [see Eq. (1)], the eigen emittances and projected rms emittances are different. The product of the eigen emittances cannot be larger than the product of projected rms emittances. The four-dimensional beam rms emittance is calculated as

$$
\varepsilon_{4 d}=\varepsilon_{1} \varepsilon_{2}=\sqrt{|C|} \leq \varepsilon_{x} \varepsilon_{y} .
$$

The coupling parameter $t$ is introduced to quantify interplane coupling as

$$
t:=\frac{\varepsilon_{x} \varepsilon_{y}}{\varepsilon_{1} \varepsilon_{2}}-1 \geq 0,
$$

and if $t$ is equal to zero, there are no interplane correlations and the projected rms emittances are equal to the eigen emittances.

\section{ROSE METHOD}

ROSE has been developed to measure the full fourdimensional transverse beam matrix of ion beams as shown in Fig. 1. It is a slit and grid combination being installed inside a rotatable vacuum chamber. In the slit and grid system of ROSE the slit has an opening width of $d_{\text {slit }}=$ $0.2 \mathrm{~mm}$ and the step width is typically $\delta \mu=0.5 \mathrm{~mm}$. Slit and grid are separated by $d=300 \mathrm{~mm}$, the wire distance is $d_{\text {wire }}=1.0 \mathrm{~mm}$, and the intermediate step number for moving the grid is $n=4$. The spacial and angular resolution of the emittance measurements is accordingly

$$
\Delta \mu:=\sqrt{\left(d_{\text {slit }}\right)^{2}+(\delta \mu)^{2}},
$$




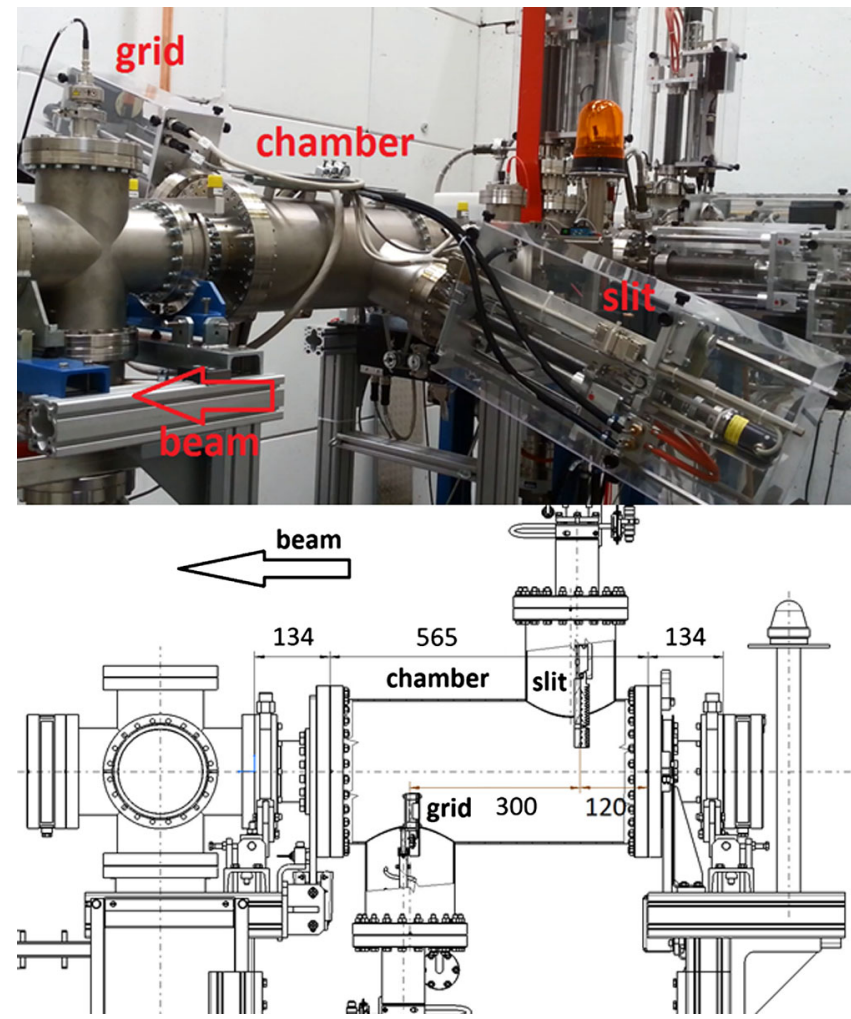

FIG. 1. Rotating system for emittance measurements: a singleplane slit and grid emittance measurement device housed in a chamber which can be rotated around the beam axis. The total length of ROSE is $833 \mathrm{~mm}$.

$$
\Delta \mu^{\prime}=\frac{\sqrt{\left(\frac{d_{\text {wire }}}{1+n}\right)^{2}+\left(d_{\text {slit }}\right)^{2}}}{d}
$$

determined to be $0.5 \mathrm{~mm} / 0.9 \mathrm{mrad}$. The emittance measurement unit can be rotated around the beam axis by a total of $270^{\circ}$ and the rotation can be done within $2 \mathrm{~min}$. One emittance measurement takes about $15 \mathrm{~min}$. For rotation no shutters need to be closed. The vacuum pressure increases from few $10^{-8}$ mbar to few $10^{-7}$ mbar during rotation. Afterward the pressure recovers within about 3 min. A detailed description of the mechanical setup can be found in [29].

ROSE has been installed at the high charge injector (HLI) section [30], as it is fed by an ECR source that provides correlated beams. It is installed as a mobile setup, i.e. the corresponding chamber may be installed at many locations along the versatile GSI beam lines. The complete beam line consists of one skew quadrupole triplet and one regular quadrupole doublet followed by the ROSE unit as shown in Fig. 2.

\section{A. Mathematical algorithms}

The transport of the beam matrix from location $i$ to location $f$ can be calculated as (see Fig. 3)

$$
C_{f}=M C_{i} M^{T}
$$

where $M$ is the transport matrix between location $i$ and location $f$

$$
M=\left[\begin{array}{llll}
m_{11} & m_{12} & m_{13} & m_{14} \\
m_{21} & m_{22} & m_{23} & m_{24} \\
m_{31} & m_{32} & m_{33} & m_{34} \\
m_{41} & m_{42} & m_{43} & m_{44}
\end{array}\right]:=\left[\begin{array}{ll}
M_{x x} & M_{x y} \\
M_{y x} & M_{y y}
\end{array}\right]
$$

As the ROSE beam line is without coupling elements if the skew quadrupole triplet is switched off, the offdiagonal submatrices of the transport matrices vanish, i.e. $M_{x y}=M_{y x}=0$. The transports $M^{a}$ or $M^{b}$ of single particle coordinates from location $i$ to location $f$ using magnet settings $a$ or $b$ are described by transfer matrices through

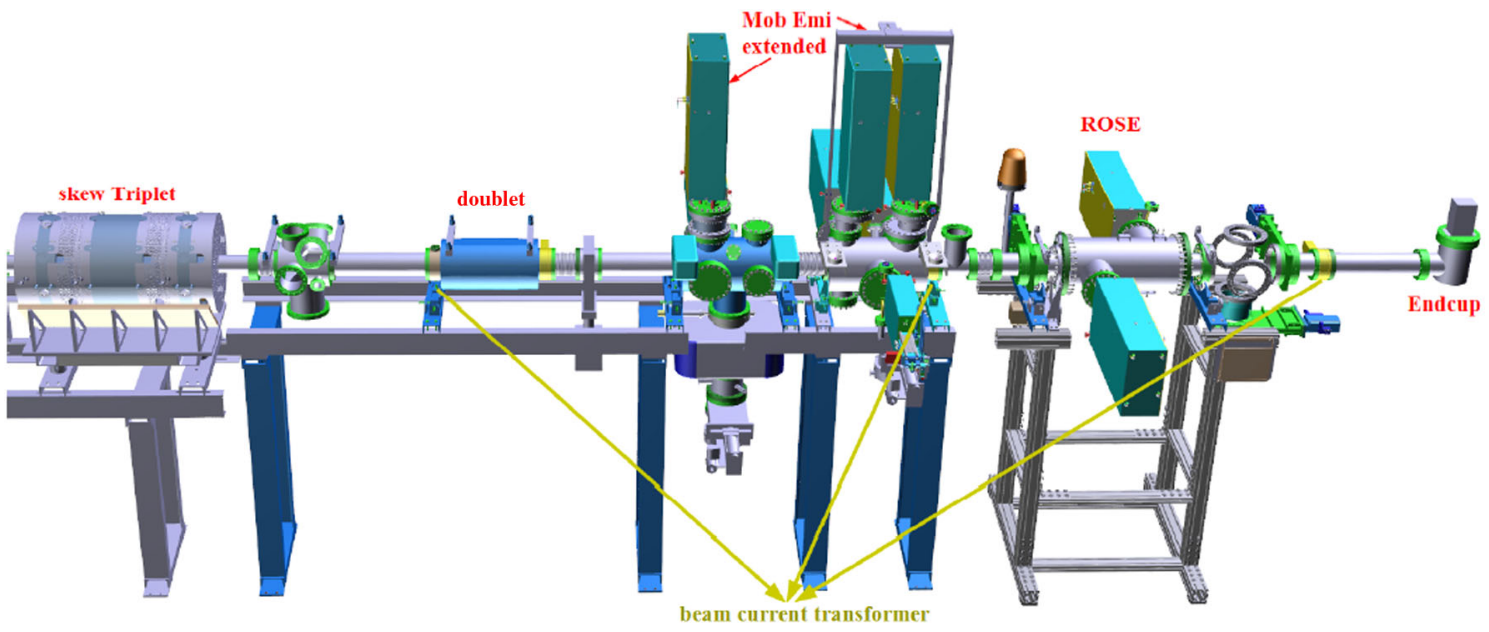

FIG. 2. Setup of the beam line with ROSE. The beam enters from the left. 


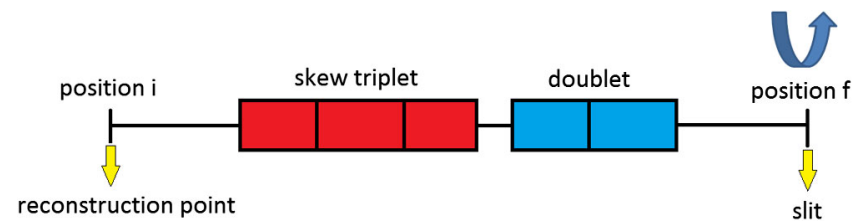

FIG. 3. Sketch of the ROSE beam line with a rotatable slit/grid emittance scanner. All beam second moments are measured at location $f$. Applying the inverted transfer matrix they are transported backwards to location $i$ of the initial and constant beam matrix $C_{i}$. Full beam transmission between location $i$ and location $f$ is required.

$$
\left[\begin{array}{c}
x \\
x^{\prime} \\
y \\
y^{\prime}
\end{array}\right]_{f}^{a, b}=\left[\begin{array}{cccc}
m_{11}^{a, b} & m_{12}^{a, b} & 0 & 0 \\
m_{21}^{a, b} & m_{22}^{a, b} & 0 & 0 \\
0 & 0 & m_{33}^{a, b} & m_{34}^{a, b} \\
0 & 0 & m_{43}^{a, b} & m_{44}^{a, b}
\end{array}\right]\left[\begin{array}{c}
x \\
x^{\prime} \\
y \\
y^{\prime}
\end{array}\right]_{i}
$$

from Eq. (12); the correlated beam second moments of the off-diagonal submatrices at location $f$ using magnet settings $a$ and $b$ can be written as

$$
\begin{aligned}
\langle x y\rangle_{f}^{a, b}= & m_{11}^{a, b} m_{33}^{a, b}\langle x y\rangle_{i}+m_{11}^{a, b} m_{34}^{a, b}\left\langle x y^{\prime}\right\rangle_{i} \\
& +m_{12}^{a, b} m_{33}^{a, b}\left\langle x^{\prime} y\right\rangle_{i}+m_{12}^{a, b} m_{34}^{a, b}\left\langle x^{\prime} y^{\prime}\right\rangle_{i},
\end{aligned}
$$

$$
\begin{aligned}
\left\langle x y^{\prime}\right\rangle_{f}^{a, b}+\left\langle x^{\prime} y\right\rangle_{f}^{a, b}= & \left(m_{11}^{a, b} m_{43}^{a, b}+m_{21}^{a, b} m_{33}^{a, b}\right)\langle x y\rangle_{i} \\
& +\left(m_{11}^{a, b} m_{44}^{a, b}+m_{21}^{a, b} m_{34}^{a, b}\right)\left\langle x y^{\prime}\right\rangle_{i} \\
& +\left(m_{12}^{a, b} m_{43}^{a, b}+m_{22}^{a, b} m_{33}^{a, b}\right)\left\langle x^{\prime} y\right\rangle_{i} \\
& +\left(m_{12}^{a, b} m_{44}^{a, b}+m_{22}^{a, b} m_{34}^{a, b}\right)\left\langle x^{\prime} y^{\prime}\right\rangle_{i}, \\
\left\langle x^{\prime} y^{\prime}\right\rangle_{f}^{a, b}= & m_{21}^{a, b} m_{43}^{a, b}\langle x y\rangle_{i}+m_{21}^{a, b} m_{44}^{a, b}\left\langle x y^{\prime}\right\rangle_{i} \\
& +m_{22}^{a, b} m_{43}^{a, b}\left\langle x^{\prime} y\right\rangle_{i}+m_{22}^{a, b} m_{44}^{a, b}\left\langle x^{\prime} y^{\prime}\right\rangle_{i} .
\end{aligned}
$$

Rotating clockwise the emittance measurement unit by $\theta$ is equivalent to rotating the beam by $-\theta$ around the beam axis. After rotation, the new particle coordinates using magnet settings $a$ or $b$ are transported by a simple rotation matrix

$$
\left[\begin{array}{l}
x \\
x^{\prime} \\
y \\
y^{\prime}
\end{array}\right]_{\theta}^{a, b}=\left[\begin{array}{cccc}
\cos \theta & 0 & \sin \theta & 0 \\
0 & \cos \theta & 0 & \sin \theta \\
-\sin \theta & 0 & \cos \theta & 0 \\
0 & -\sin \theta & 0 & \cos \theta
\end{array}\right]\left[\begin{array}{l}
x \\
x^{\prime} \\
y \\
y^{\prime}
\end{array}\right]_{f}^{a, b} .
$$

According to Eq. (16) horizontal second moments after rotation using magnet settings $a$ and $b$ are expressed as

$$
\begin{aligned}
\langle x x\rangle_{\theta}^{a, b}= & \cos ^{2} \theta\langle x x\rangle_{f}^{a, b}+2 \sin \theta \cos \theta\langle x y\rangle_{f}^{a, b} \\
& +\sin ^{2} \theta\langle y y\rangle_{f}^{a, b}, \\
\left\langle x x^{\prime}\right\rangle_{\theta}^{a, b}= & \cos ^{2} \theta\left\langle x x^{\prime}\right\rangle_{f}^{a, b}+\sin \theta \cos \theta\left\langle x y^{\prime}\right\rangle_{f}^{a, b} \\
& +\sin \theta \cos \theta\left\langle x^{\prime} y\right\rangle_{f}^{a, b}+\sin ^{2} \theta\left\langle y y^{\prime}\right\rangle_{f}^{a, b}, \\
\left\langle x^{\prime} x^{\prime}\right\rangle_{\theta}^{a, b}= & \cos ^{2} \theta\left\langle x^{\prime} x^{\prime}\right\rangle_{f}^{a, b}+2 \sin \theta \cos \theta\left\langle x^{\prime} y^{\prime}\right\rangle_{f}^{a, b} \\
+ & \sin ^{2} \theta\left\langle y^{\prime} y^{\prime}\right\rangle_{f}^{a, b} .
\end{aligned}
$$

All elements of the transport matrices $M_{x x}^{a, b}$ and $M_{y y}^{a, b}$ are known from magnet settings. The second moments $\langle x x\rangle_{f}^{a, b}$, $\left\langle x x^{\prime}\right\rangle_{f}^{a, b},\left\langle x^{\prime} x^{\prime}\right\rangle_{f}^{a, b},\langle y y\rangle_{f}^{a, b},\left\langle y y^{\prime}\right\rangle_{f}^{a, b}$, and $\left\langle y^{\prime} y^{\prime}\right\rangle_{f}^{a, b}$ before rotation and $\langle x x\rangle_{\theta}^{a, b},\left\langle x x^{\prime}\right\rangle_{\theta}^{a, b}$, and $\left\langle x^{\prime} x^{\prime}\right\rangle_{\theta}^{a, b}$ after rotation can be measured. Combining Eq. (13) to Eq. (19), the solution of the searched coupled matrix elements at location $i$ can be summarized to a set of linear equations

$\left\{\begin{array}{l}\Gamma_{11}\langle x y\rangle_{i}+\Gamma_{12}\left\langle x y^{\prime}\right\rangle_{i}+\Gamma_{13}\left\langle x^{\prime} y\right\rangle_{i}+\Gamma_{14}\left\langle x^{\prime} y^{\prime}\right\rangle_{i}=\Lambda_{1} \\ \Gamma_{21}\langle x y\rangle_{i}+\Gamma_{22}\left\langle x y^{\prime}\right\rangle_{i}+\Gamma_{23}\left\langle x^{\prime} y\right\rangle_{i}+\Gamma_{24}\left\langle x^{\prime} y^{\prime}\right\rangle_{i}=\Lambda_{2} \\ \Gamma_{31}\langle x y\rangle_{i}+\Gamma_{32}\left\langle x y^{\prime}\right\rangle_{i}+\Gamma_{33}\left\langle x^{\prime} y\right\rangle_{i}+\Gamma_{34}\left\langle x^{\prime} y^{\prime}\right\rangle_{i}=\Lambda_{3} \\ \Gamma_{41}\langle x y\rangle_{i}+\Gamma_{42}\left\langle x y^{\prime}\right\rangle_{i}+\Gamma_{43}\left\langle x^{\prime} y\right\rangle_{i}+\Gamma_{44}\left\langle x^{\prime} y^{\prime}\right\rangle_{i}=\Lambda_{4} \\ \Gamma_{51}\langle x y\rangle_{i}+\Gamma_{52}\left\langle x y^{\prime}\right\rangle_{i}+\Gamma_{53}\left\langle x^{\prime} y\right\rangle_{i}+\Gamma_{54}\left\langle x^{\prime} y^{\prime}\right\rangle_{i}=\Lambda_{5} \\ \Gamma_{61}\langle x y\rangle_{i}+\Gamma_{62}\left\langle x y^{\prime}\right\rangle_{i}+\Gamma_{63}\left\langle x^{\prime} y\right\rangle_{i}+\Gamma_{64}\left\langle x^{\prime} y^{\prime}\right\rangle_{i}=\Lambda_{6}\end{array}\right.$

with

$$
\begin{array}{cl}
\Gamma_{11}=m_{11}^{a} m_{33}^{a}, & \Gamma_{12}=m_{11}^{a} m_{34}^{a}, \\
\Gamma_{13}=m_{12}^{a} m_{33}^{a}, & \Gamma_{14}=m_{12}^{a} m_{34}^{a}, \\
\Gamma_{21}=m_{11}^{a} m_{43}^{a}+m_{21}^{a} m_{33}^{a}, & \Gamma_{22}=m_{11}^{a} m_{44}^{a}+m_{21}^{a} m_{34}^{a}, \\
\Gamma_{23}=m_{12}^{a} m_{43}^{a}+m_{22}^{a} m_{33}^{a}, & \Gamma_{24}=m_{12}^{a} m_{44}^{a}+m_{22}^{a} m_{34}^{a},
\end{array}
$$

$$
\begin{array}{ll}
\Gamma_{31}=m_{21}^{a} m_{43}^{a}, & \Gamma_{32}=m_{21}^{a} m_{44}^{a}, \\
\Gamma_{33}=m_{22}^{a} m_{43}^{a}, & \Gamma_{34}=m_{22}^{a} m_{44}^{a},
\end{array}
$$

and with the same procedure for setting $b$

$$
\begin{aligned}
\Gamma_{41}=m_{11}^{b} m_{33}^{b}, & \Gamma_{42}=m_{11}^{b} m_{34}^{b}, \\
\Gamma_{43}=m_{12}^{b} m_{33}^{b}, & \Gamma_{44}=m_{12}^{b} m_{34}^{b}, \\
\Gamma_{51}=m_{11}^{b} m_{43}^{b}+m_{21}^{b} m_{33}^{b}, & \Gamma_{52}=m_{11}^{b} m_{44}^{b}+m_{21}^{b} m_{34}^{b}, \\
\Gamma_{53}=m_{12}^{b} m_{43}^{b}+m_{22}^{b} m_{33}^{b}, & \Gamma_{54}=m_{12}^{b} m_{44}^{b}+m_{22}^{b} m_{34}^{b},
\end{aligned}
$$




$$
\begin{array}{ll}
\Gamma_{61}=m_{21}^{b} m_{43}^{b}, & \Gamma_{62}=m_{21}^{b} m_{44}^{b}, \\
\Gamma_{63}=m_{22}^{b} m_{43}^{b}, & \Gamma_{64}=m_{22}^{b} m_{44}^{b},
\end{array}
$$

and finally

$$
\begin{array}{lll}
\Lambda_{1}=\langle x y\rangle_{f}^{a}, & \Lambda_{2}=\left\langle x y^{\prime}\right\rangle_{f}^{a}+\left\langle x^{\prime} y\right\rangle_{f}^{a}, & \Lambda_{3}=\left\langle x^{\prime} y^{\prime}\right\rangle_{f}^{a}, \\
\Lambda_{4}=\langle x y\rangle_{f}^{b}, & \Lambda_{5}=\left\langle x y^{\prime}\right\rangle_{f}^{b}+\left\langle x^{\prime} y\right\rangle_{f}^{b}, & \Lambda_{6}=\left\langle x^{\prime} y^{\prime}\right\rangle_{f}^{b} .
\end{array}
$$

Our goal is to determine the unknown coefficients $\langle x y\rangle_{i}$, $\left\langle x y^{\prime}\right\rangle_{i},\left\langle x^{\prime} y\right\rangle_{i}$, and $\left\langle x^{\prime} y^{\prime}\right\rangle_{i}$ such that the linear model is a best fit to the observed data $\Lambda_{1}, \Lambda_{2}, \ldots \Lambda_{6}$. Consider a system of $n=6$ linear equations with $k=4$ variables [see Eq. (20)] being an overdetermined linear system

$$
\left[\begin{array}{llll}
\Gamma_{11} & \Gamma_{12} & \Gamma_{13} & \Gamma_{14} \\
\Gamma_{21} & \Gamma_{22} & \Gamma_{23} & \Gamma_{24} \\
\Gamma_{31} & \Gamma_{32} & \Gamma_{33} & \Gamma_{34} \\
\Gamma_{41} & \Gamma_{42} & \Gamma_{43} & \Gamma_{44} \\
\Gamma_{51} & \Gamma_{52} & \Gamma_{53} & \Gamma_{54} \\
\Gamma_{61} & \Gamma_{62} & \Gamma_{63} & \Gamma_{64}
\end{array}\right]\left[\begin{array}{c}
\langle x y\rangle_{i} \\
\left\langle x y^{\prime}\right\rangle_{i} \\
\left\langle x^{\prime} y\right\rangle_{i} \\
\left\langle x^{\prime} y^{\prime}\right\rangle_{i}
\end{array}\right]=\left[\begin{array}{c}
\Lambda_{1} \\
\Lambda_{2} \\
\Lambda_{3} \\
\Lambda_{4} \\
\Lambda_{5} \\
\Lambda_{6}
\end{array}\right] .
$$

A system of equations is considered overdetermined if there are more equations than unknowns. The method of ordinary least squares can be used to find an approximate solution to overdetermined systems and the solution of Eq. (29) can be written with the normal equations

$$
\left[\begin{array}{c}
\langle x y\rangle_{i} \\
\left\langle x y^{\prime}\right\rangle_{i} \\
\left\langle x^{\prime} y\right\rangle_{i} \\
\left\langle x^{\prime} y^{\prime}\right\rangle_{i}
\end{array}\right]=\left(\Gamma^{T} \Gamma\right)^{-1} \Gamma^{T} \Lambda,
$$

where

$$
\Gamma=\left[\begin{array}{llll}
\Gamma_{11} & \Gamma_{12} & \Gamma_{13} & \Gamma_{14} \\
\Gamma_{21} & \Gamma_{22} & \Gamma_{23} & \Gamma_{24} \\
\Gamma_{31} & \Gamma_{32} & \Gamma_{33} & \Gamma_{34} \\
\Gamma_{41} & \Gamma_{42} & \Gamma_{43} & \Gamma_{44} \\
\Gamma_{51} & \Gamma_{52} & \Gamma_{53} & \Gamma_{54} \\
\Gamma_{61} & \Gamma_{62} & \Gamma_{63} & \Gamma_{64}
\end{array}\right], \quad \Lambda=\left[\begin{array}{c}
\Lambda_{1} \\
\Lambda_{2} \\
\Lambda_{3} \\
\Lambda_{4} \\
\Lambda_{5} \\
\Lambda_{6}
\end{array}\right]
$$

provided $\left(\Gamma^{T} \Gamma\right)^{-1}$ exists (the $k=4$ columns of $\Gamma$ are linearly independent). With this formula an approximate solution is found if no exact solution exists, and it gives an exact solution if one does exist.

\section{B. Measurement procedure}

Projected rms-emittance measurements can be performed at various angles, i.e. $0^{\circ}, 90^{\circ}$, and $\Theta^{\circ}$ (any angle which is not equivalent to $0^{\circ}$ or $90^{\circ}$ ) to reconstruct the full four-dimensional beam matrix $C_{i}$. Rotation by $0^{\circ} / 90^{\circ}$ will just measure the usual uncoupled second moments. Minimum of four, but more reliable six measurements is sufficient to measure the complete four-dimensional beam matrix: (i) measurements at $\theta=0^{\circ}$ with optics $a$ (and $b$ ). (ii) measurements at $\theta=90^{\circ}$ with optics $a$ (and $b$ ). (iii) measurements at $\theta=\Theta^{\circ}$ with optics $a$ and $b$.

If just four measurements are applied to evaluate the full beam matrix at location $i$, the uncoupled second moments for setting $b$ at location $f$, i.e. $\langle x x\rangle_{f}^{b},\left\langle x x^{\prime}\right\rangle_{f}^{b}$, and $\left\langle x^{\prime} x^{\prime}\right\rangle_{f}^{b}$ are calculated from the final uncoupled second moments for setting $a$, i.e. $\langle x x\rangle_{f}^{a},\left\langle x x^{\prime}\right\rangle_{f}^{a}$, and $\left\langle x^{\prime} x^{\prime}\right\rangle_{f}^{a}$ using the transport matrices $M_{x x}^{a}$ and $M_{x x}^{b}$.

The four measurements (projected rms emittances and Twiss parameters) and their deliverables are as follows: (i) $\theta=0^{\circ}$, magnet setting $a$ delivers $\langle x x\rangle_{f}^{a},\left\langle x x^{\prime}\right\rangle_{f}^{a}$, and $\left\langle x^{\prime} x^{\prime}\right\rangle_{f}^{a}$. (ii) $\theta=90^{\circ}$, magnet setting $a$ delivers $\langle y y\rangle_{f}^{a}$, $\left\langle y y^{\prime}\right\rangle_{f}^{a}$, and $\left\langle y^{\prime} y^{\prime}\right\rangle_{f}^{a}$. (iii) $\theta=\Theta^{\circ}$, magnet setting $a$ delivers $\langle x x\rangle_{\theta}^{a},\left\langle x x^{\prime}\right\rangle_{\theta}^{a}$, and $\left\langle x^{\prime} x^{\prime}\right\rangle_{\theta}^{a}$. (iv) $\theta=\Theta^{\circ}$, magnet setting $b$ delivers $\langle x x\rangle_{\theta}^{b},\left\langle x x^{\prime}\right\rangle_{\theta}^{b}$, and $\left\langle x^{\prime} x^{\prime}\right\rangle_{\theta}^{b}$.

From step (i) the uncorrelated second moments $\langle x x\rangle_{i}$, $\left\langle x x^{\prime}\right\rangle_{i}$, and $\left\langle x^{\prime} x^{\prime}\right\rangle_{i}$ are obtained at location $i$ by simple back transformation through inversion of Eq. (12). From step (ii) the uncorrelated beam moments $\langle y y\rangle_{i},\left\langle y y^{\prime}\right\rangle_{i}$, and $\left\langle y^{\prime} y^{\prime}\right\rangle_{i}$ are obtained at location $i$ in the same way. Steps (iii) and (iv) deliver $\langle x y\rangle_{f}^{a, b},\left\langle x y^{\prime}\right\rangle_{f}^{a, b}+\left\langle x^{\prime} y\right\rangle_{f}^{a, b}$, and $\left\langle x^{\prime} y^{\prime}\right\rangle_{f}^{a, b}$ at location $f$ [Eq. (13) to Eq. (15)]. Finally, Eq. (30) determines the correlated second moments $\langle x y\rangle_{i},\left\langle x y^{\prime}\right\rangle_{i},\left\langle x^{\prime} y\right\rangle_{i}$, and $\left\langle x^{\prime} y^{\prime}\right\rangle_{i}$ at location $i$. The four-dimensional second moments beam matrix is then finally reconstructed at location $i$ from four measurements.

If six measurements are applied, the uncoupled second moments for setting $b$ at location $f$ are measured directly.

\section{Minimizing the measurement errors}

The vector $\Lambda$ [see Eq. (30)] is sensitive to the emittance measurements at location $f$. During emittance measurements, finite grid bin resolution and background noise have influence on the measured second moments. The typical error of directly measured second moments is about $\pm 10 \%$. These errors enter into the inversion of Eq. (30).

In general a nonsquare matrix $\Gamma$ has no inverse in the usual sense. But if $\Gamma$ has full rank, a pseudoinverse can be defined as [31]

$$
\Gamma^{\dagger}=\left(\Gamma^{T} \Gamma\right)^{-1} \Gamma^{T}
$$

and the condition number of this overdetermined linear system is defined as 


$$
\kappa(\Gamma):=\|\Gamma\|_{2}\left\|\Gamma^{\dagger}\right\|_{2},
$$

being larger than or equal to 1.0. The Frobenius norm, or the Hilbert-Schmidt norm of $\Gamma$ and $\Gamma^{\dagger}$ are defined as

$$
\|\Gamma\|_{2}:=\sqrt{\sum_{i=1}^{n} \sum_{j=1}^{k}\left(\Gamma_{i, j}\right)^{2}}, \quad\left\|\Gamma^{\dagger}\right\|_{2}:=\sqrt{\sum_{i=1}^{k} \sum_{j=1}^{n}\left(\Gamma_{i, j}^{\dagger}\right)^{2} .}
$$

The condition number associated with the linear equations [see Eq. (29)] is a measure for how well conditioned the matrix is. If the condition number is large, even a small error in emittance measurements may lead to radically different results for the beam coupling parameter evaluations. On the other hand, if the condition number is small the error in evaluation will not exceed notably the error in emittance measurements. The numerical stability (degeneration of the system) is better if the condition number is small. Well-conditioned matrices have condition numbers which are closed to 1.0.

We summarize that in order to obtain reliable evaluation results a four-dimensional emittance measurement needs: (i) one reference emittance measurement with $100 \%$ transmission efficiency between location $i$ and location $f$ to obtain projected beam parameters at location $i$ (on-diagonal section of beam matrix of $C_{i}$ ). (ii) all quadrupoles varied numerically in a brute-force method in order to check each setting for full transmission efficiency from location $i$ to location $f$, and for reasonable beam sizes on slit and grid ( $2 \mathrm{~mm}<\sigma_{\text {rms }}<5 \mathrm{~mm}$ in our case). In the plane spanned by the two quadrupole gradients these settings form finite areas. We refer to these areas as safety islands in the following. (iii) All settings from safety islands are combined to determine combinations of two settings $a$ and $b$ corresponding to a low condition number.

\section{MEASUREMENTS AND EVALUATIONS}

In a first measurement the projected rms emittances and Twiss parameters at the exit of the ROSE beam line were measured as listed in Table I. A beam of ${ }^{83} \mathrm{Kr}^{13+}$ at $1.4 \mathrm{MeV} / \mathrm{u}$ has been used, the beam intensity through the ROSE was about $20 e \mu \mathrm{A}$, and space-charge effects can be neglected in this case. The skew triplet and normal doublet were switched off and the transmission through the

TABLE I. Projected rms emittances and Twiss parameters measured at ROSE for ${ }^{83} \mathrm{Kr}^{13+}$ at $1.4 \mathrm{MeV} / \mathrm{u}$ (reference measurements).

\begin{tabular}{lccc}
\hline \hline Rotation angle & $\alpha_{\mathrm{rms}}$ & $\beta_{\mathrm{rms}}[\mathrm{m} / \mathrm{rad}]$ & $\varepsilon_{\mathrm{rms}}[\mathrm{mm} \mathrm{mrad}]$ \\
\hline $0^{\circ}$ & -2.92 & 12.92 & 2.03 \\
$90^{\circ}$ & -1.63 & 10.52 & 2.43 \\
\hline \hline
\end{tabular}

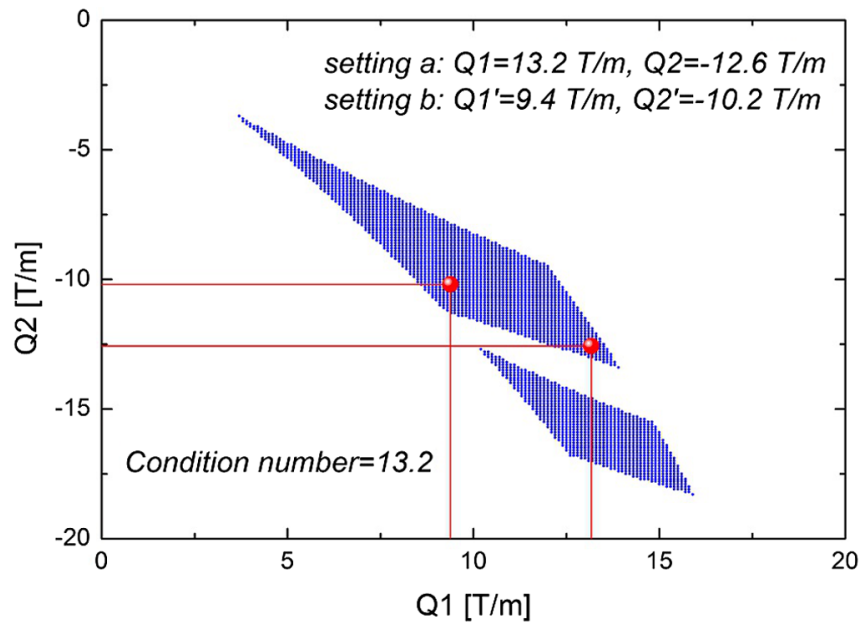

FIG. 4. Safety islands (blue dots) and the selected doublet settings (red dots) applied during the measurements.

setup was $100 \%$. Uncoupled second moments at location $i$ were obtained from $0^{\circ} / 90^{\circ}$ measurements at location $f$ to reconstruct the on-diagonal section of $C_{i}$. In order to match reasonable beam sizes on the slit and gird locations and to assure full transmission, the strengths of $Q_{1}$ and $Q_{2}$ were varied numerically to check all available doublet settings, i.e. the safety islands including all reasonable doublet settings were defined. Combining two settings of the doublet $\left(Q_{1}\right.$ and $Q_{2}, Q_{1}{ }^{\prime}$ and $Q_{2}^{\prime}$ ) from the safety islands, the corresponding condition number of the matrix $\Gamma$ was calculated using Eq. (33). Since there are $N$ doublet settings inside the safety islands, $N^{2}$ combinations of two doublet settings were obtained. Finally, the combination of doublet settings $a$ and $b$ with minimum condition number $\kappa(\Gamma)$ is applied.

The doublet setting $a: Q 1 / Q 2=13.2 /-12.6 \mathrm{~T} / \mathrm{m}$ and setting $b: Q 1^{\prime} / Q 2^{\prime}=9.4 /-10.2 \mathrm{~T} / \mathrm{m}$ were selected as they provide the condition number 13.2. The condition number is determined by the transfer matrix from position $i$ to position $f$, i.e. it does not depend on the number of measurements [four or six, see Eq. (33)]. The safety islands and the selected doublet settings are plotted in Fig. 4.

\section{A. Beam with low coupling}

Measurements at $0^{\circ}, 90^{\circ}$, and $-30^{\circ}$ using settings $a$ and $b$ with the skew triplet being switched off were done. The

TABLE II. Measured projected rms emittances and Twiss parameters at the exit of the ROSE beam line with the skew triplet being switched off.

\begin{tabular}{lcrcc}
\hline \hline Rotation angle & Setting & \multicolumn{1}{c}{$\alpha_{\mathrm{rms}}$} & $\beta_{\mathrm{rms}}[\mathrm{m} / \mathrm{rad}]$ & $\varepsilon_{\mathrm{rms}}[\mathrm{mm} \mathrm{mrad}]$ \\
\hline $0^{\circ}$ & $\mathrm{a}$ & 0.25 & 3.97 & 1.93 \\
$0^{\circ}$ & $\mathrm{b}$ & -0.01 & 4.53 & 1.93 \\
$90^{\circ}$ & $\mathrm{a}$ & -1.84 & 3.97 & 2.45 \\
$90^{\circ}$ & $\mathrm{b}$ & -1.06 & 6.63 & 2.81 \\
$-30^{\circ}$ & $\mathrm{a}$ & -0.41 & 3.78 & 2.27 \\
$-30^{\circ}$ & $\mathrm{b}$ & -0.57 & 4.76 & 2.16 \\
\hline \hline
\end{tabular}



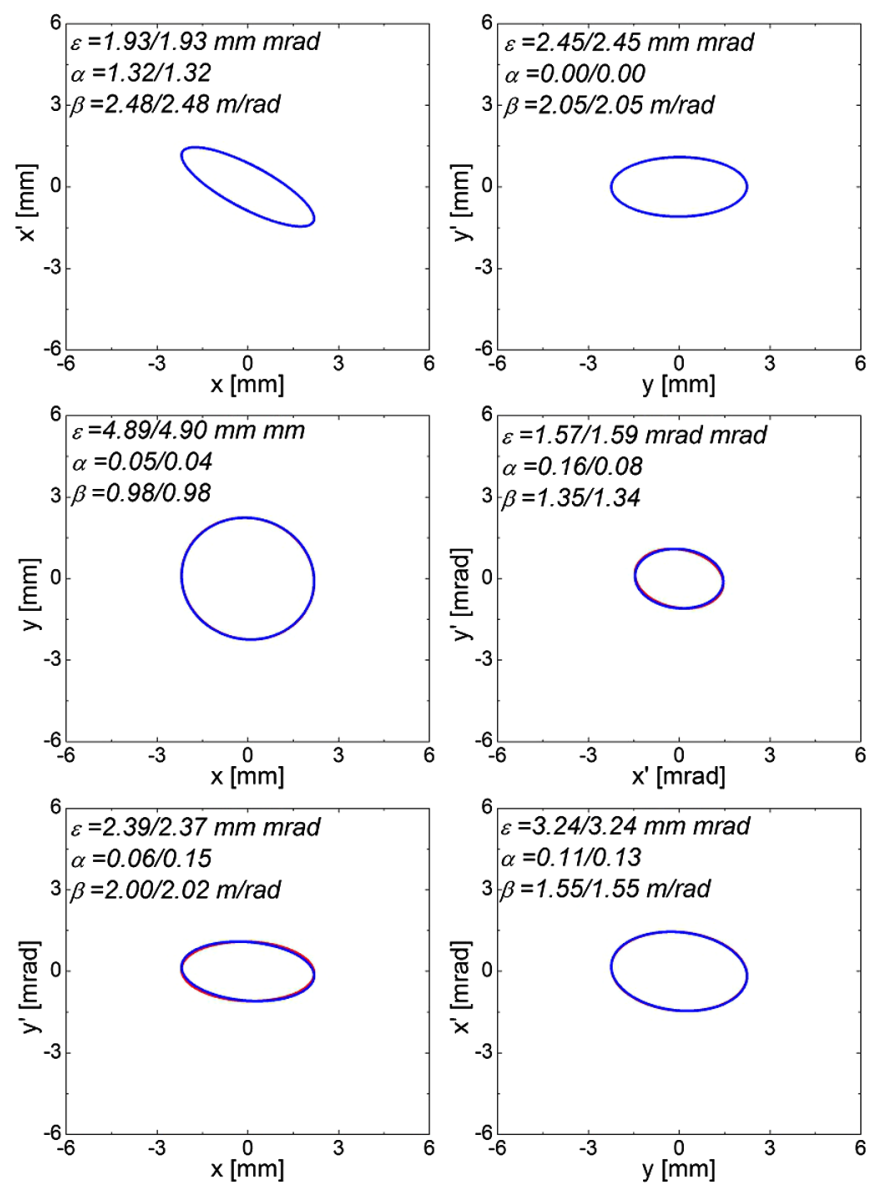

FIG. 5. Projected rms ellipses from measurements applying four or six measurements with the skew triplet being switched off. Red and blue ellipses indicate the beam matrices $\hat{C}(4)$ and $\hat{C}(6)$. The projected rms emittances and the Twiss parameters are indicated. The two matrices produce almost identical rms ellipses and feature similar eigen emittances and coupling parameters.

measured Twiss parameters together with the projected rms emittances are listed in Table II. Two evaluations using four or six measurements were done independently for comparison.

The beam second moments matrix $\hat{C}(4)$ applying four measurements at location $i$ with skews off is calculated as (in units of $\mathrm{mm}$ and $\mathrm{mrad}$ )

$$
\hat{C}(4)=\left[\begin{array}{cccc}
4.79 & -2.54 & -0.14 & -0.17 \\
-2.54 & 2.12 & -0.29 & -0.22 \\
-0.14 & -0.29 & 5.02 & 0.02 \\
-0.17 & -0.22 & 0.02 & 1.20
\end{array}\right]
$$

The beam second moments matrix $\hat{C}(6)$ applying six measurements at location $i$ with skews off is calculated as (in units of $\mathrm{mm}$ and $\mathrm{mrad}$ )
TABLE III. Measured projected rms-emittances and Twiss parameters at the exit of the ROSE beam line with the skew triplet being switched on.

\begin{tabular}{lcrcc}
\hline \hline Rotation angle & Setting & \multicolumn{1}{c}{$\alpha_{\text {rms }}$} & $\beta_{\text {rms }}[\mathrm{m} / \mathrm{rad}]$ & $\varepsilon_{\text {rms }}[\mathrm{mm} \mathrm{mrad}]$ \\
\hline $0^{\circ}$ & $\mathrm{b}$ & -0.14 & 4.61 & 3.15 \\
$0^{\circ}$ & $\mathrm{a}$ & 0.10 & 3.95 & 3.13 \\
$90^{\circ}$ & $\mathrm{b}$ & -2.45 & 8.79 & 3.41 \\
$90^{\circ}$ & $\mathrm{a}$ & -2.69 & 7.35 & 3.33 \\
$30^{\circ}$ & $\mathrm{b}$ & -0.55 & 2.25 & 3.20 \\
$30^{\circ}$ & $\mathrm{a}$ & -0.80 & 2.68 & 4.67 \\
\hline \hline$\hat{C}(6)=$ & {$\left[\begin{array}{ccccc}4.79 & -2.54 & -0.10 & -0.44 \\
-2.54 & 2.12 & -0.25 & -0.12 \\
-0.10 & -0.25 & 5.02 & 0.02 \\
-0.44 & -0.12 & 0.02 & 1.20\end{array}\right] . \quad(36)$}
\end{tabular}

Evaluation of the two eigen emittances of $\hat{C}(4)$ and $\hat{C}(6)$ reveals $\varepsilon_{1}=2.63$ and $2.63 \mathrm{~mm} \mathrm{mrad}$ and $\varepsilon_{2}=1.66$ and $1.61 \mathrm{~mm} \mathrm{mrad}$. The corresponding coupling parameters $t$ are 0.08 and 0.11 . Both evaluations produce similar eigen emittances and coupling parameters. The rms ellipses of the matrices $\hat{C}(4)$ and $\hat{C}(6)$ in the projections are shown in Fig. 5.

\section{B. Beam with large coupling}

As no significant initial correlations were found to be present in the HLI beam, the skew triplet was switched on in order to create correlation. Measurements were done at $0^{\circ}, 90^{\circ}$, and $30^{\circ}$ using settings $a$ and $b$. The Twiss parameters together with the projected rms emittances are listed in Table III.

The beam second moments matrix $\tilde{C}(4)$ at location $i$ applying four measurements with skews on is calculated as (in units of $\mathrm{mm}$ and $\mathrm{mrad}$ )

$$
\tilde{C}(4)=\left[\begin{array}{cccc}
8.57 & -4.34 & -3.28 & -1.10 \\
-4.34 & 3.35 & -0.74 & 1.52 \\
-3.28 & -0.74 & 11.20 & -3.05 \\
-1.10 & 1.52 & -3.05 & 1.87
\end{array}\right] \text {. }
$$

The beam second moments matrix $\tilde{C}(6)$ at location $i$ applying six measurements with skews on is calculated as (in units of $\mathrm{mm}$ and $\mathrm{mrad}$ )

$$
\tilde{C}(6)=\left[\begin{array}{cccc}
8.57 & -4.34 & -3.83 & -1.15 \\
-4.34 & 3.35 & -0.54 & 1.52 \\
-3.83 & -0.54 & 11.20 & -3.05 \\
-1.15 & 1.52 & -3.05 & 1.87
\end{array}\right] \text {. }
$$

Evaluation of the two eigen emittances of $\tilde{C}(4)$ and $\tilde{C}(6)$ reveals $\varepsilon_{1}=2.40$ and $2.47 \mathrm{~mm} \mathrm{mrad}$ and $\varepsilon_{2}=2.04$ and 

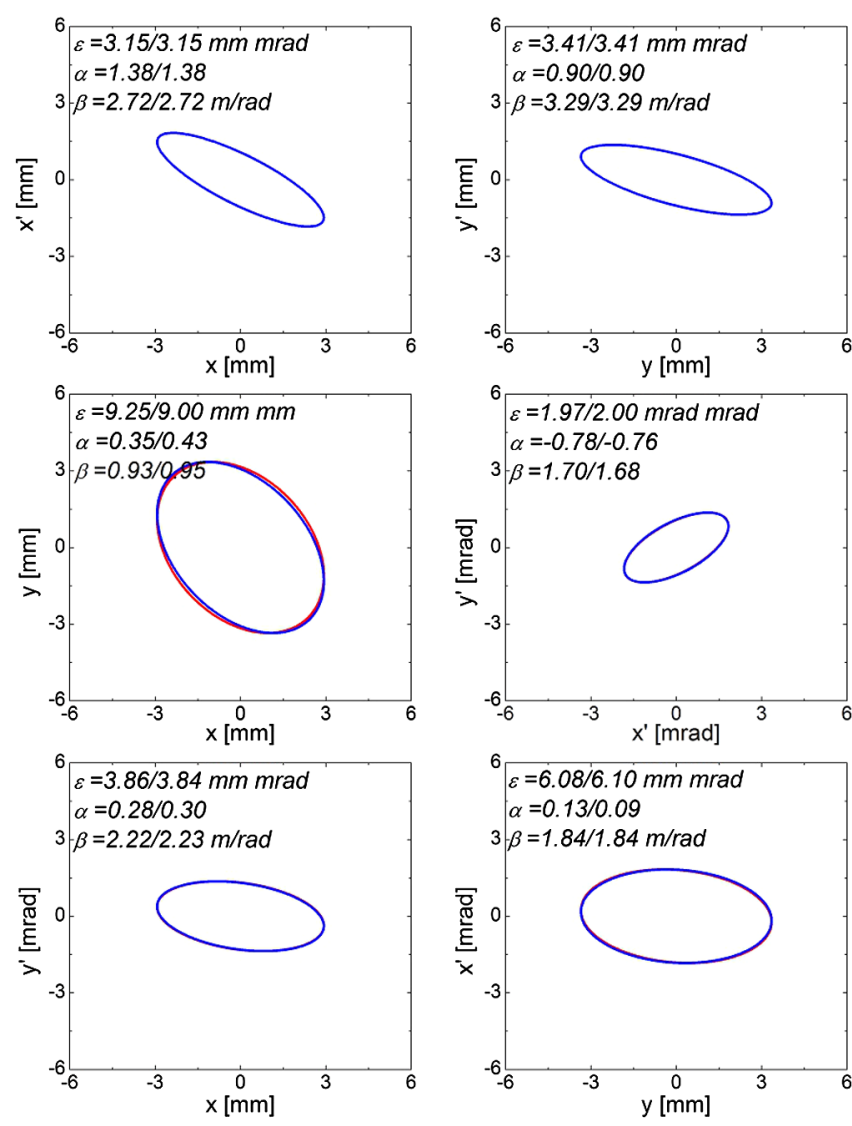

FIG. 6. Projected rms ellipses from measurements applying four or six measurements with the skew triplet being switched on. Red and blue ellipses indicate the beam matrices $\tilde{C}(4)$ and $\tilde{C}(6)$. The projected rms emittances and the Twiss parameters are indicated. According to the rms ellipses in the projections, the rms ellipses look very similar but feature different eigen emittances and coupling parameters.

$1.58 \mathrm{~mm} \mathrm{mrad}$. The corresponding coupling parameters $t$ are 1.19 and 1.74 . The beam is significantly coupled. Comparing the beam matrices $\tilde{C}(4)$ and $\tilde{C}(6)$, the difference between their larger eigen emittances is small but the smaller eigen emittances are different. The projected rms ellipses of these matrices are shown in Fig. 6. According to the rms ellipses in the projections, the rms ellipses look very similar but feature different eigen emittances and coupling parameters.

\section{Error analysis of couplings and eigen emittances}

For emittance measurement results of ROSE (slit and grid method), finite grid bin resolution and background noise had influence on measured second moments. Measured second moments on the projected rms emittance had a typical maximum error of approximately $\pm 10 \%$. In order to evaluate the errors mentioned above, we use a statistical method in which the error values of each measured second moments are normally generated within given maximum values (three times of standard deviation is equal to the maximum error: $\pm 10 \%$ ). The influences of these errors on the final coupled second moments applying four and six measurements are calculated and the corresponding frequency accounts are shown in Figs. 7 and 8 together with Gaussian fits. In statistics, a frequency count is a measure of the number of times that an event occurs. The relative frequency (or empirical probability) of an event refers to the absolute frequency normalized to the total number of events

$$
f=\frac{n}{N},
$$

where the parameters $n$ and $N$ are defined as subgroup and total frequency count. All the relative frequencies add up to $100 \%$.

Comparison of the coupled second moments with errors, Figs. 7 and 8, reveals that the mean coupled second moments are practically equal [Eqs. (35) to (38)] to the measured ones. The corresponding projected rms emittances and Twiss parameters with errors have been analyzed as well and are listed in Appendix A. The influence of these errors on the eigen emittances applying four and six measurements is calculated and the corresponding frequency accounts are shown in Figs. 9 and 10 . Comparison of the eigen emittances with and without errors reveals that the rms error span covers the values without errors if the beam is almost uncoupled; see Fig. 9. If the beam is strong correlated, see Fig. 10, the rms error span still covers the values without errors for three of the four eigen emittances.

The larger uncertainty on the measured eigen emittances for a beam inhabiting considerable correlations is already known from conventional rms-emittance measurements in one single plane (see Appendix B). The uncertainty of the measured single plane emittance is larger if the beam is strongly convergent or divergent, i.e. if it is correlated. This is just from the fact that the final observable, i.e. the emittance, is calculated from a difference [see Eq. (2)] between measured quantities. Differences are much more prone to errors from their constituents as sums or products. This sensitivity known from single-plane emittance measurements occurs in four-dimensional measurements as well, as the eigen emittances, especially $\varepsilon_{2}$, are also calculated from differences of measured quantities [Eq. (4)]. Therefore a way to reduce the error on the measured eigen emittances is to reduce the beam correlations prior to the measurements. This method has been applied successfully for single-plane measurements. In order to apply it to four-dimensional emittance measurement it needs to be demonstrated that the measured data are sufficiently accurate to perform this reduction of correlations.

\section{DECOUPLING PROSPECT ANALYSIS}

Any arbitrary beam line including at least three interplane coupling elements may serve to remove all interplane 


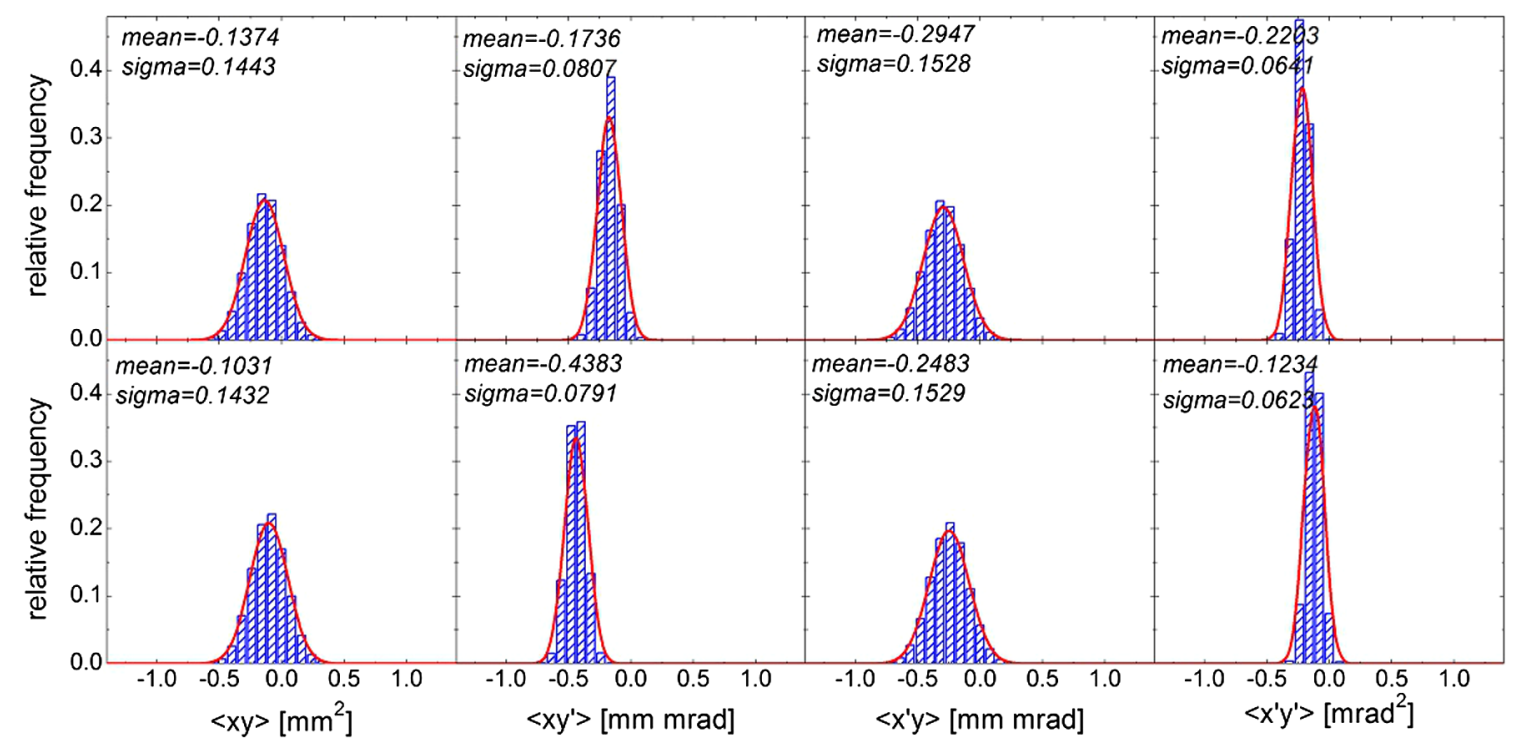

FIG. 7. Relative frequencies of the coupled second moments with skews off, the upper and lower pictures indicate the results with errors applying four and six measurements, respectively. Blue columns indicate the coupled second moments with errors and red solid lines indicate fits using Gaussian functions. The modified coupled second moments are evaluated as $\langle x y\rangle=$ $-0.14 \pm 0.14 /-0.10 \pm 0.14 \mathrm{~mm}^{2},\left\langle x y^{\prime}\right\rangle=-0.17 \pm 0.08 /-0.44 \pm 0.08 \mathrm{~mm} \mathrm{mrad},\left\langle x^{\prime} y\right\rangle=-0.29 \pm 0.15 /-0.25 \pm 0.15 \mathrm{~mm} \mathrm{mrad}$, and $\left\langle x^{\prime} y^{\prime}\right\rangle=-0.22 \pm 0.06 /-0.12 \pm 0.06 \mathrm{mrad}^{2}$.

correlations. Here a beam line composed of two normal triplets which are separated by a skew triplet is chosen. If this beam line is set to decouple the beam matrix $\tilde{C}(4)$ calculated from four measurements of the large coupling case, the corresponding decoupling transport matrix $R(4)$ is determined from the required gradients as (in units of $\mathrm{mm}$

$$
R(4)=\left[\begin{array}{cccc}
-1.0739 & -2.5538 & -0.3601 & -0.8825 \\
0.5100 & 0.4121 & 0.3255 & 0.4084 \\
-0.4128 & -1.8553 & -0.1854 & 2.1513 \\
0.1740 & 0.4424 & -0.3239 & -0.8710
\end{array}\right]
$$
and mrad) and the decoupled second moments matrix gets

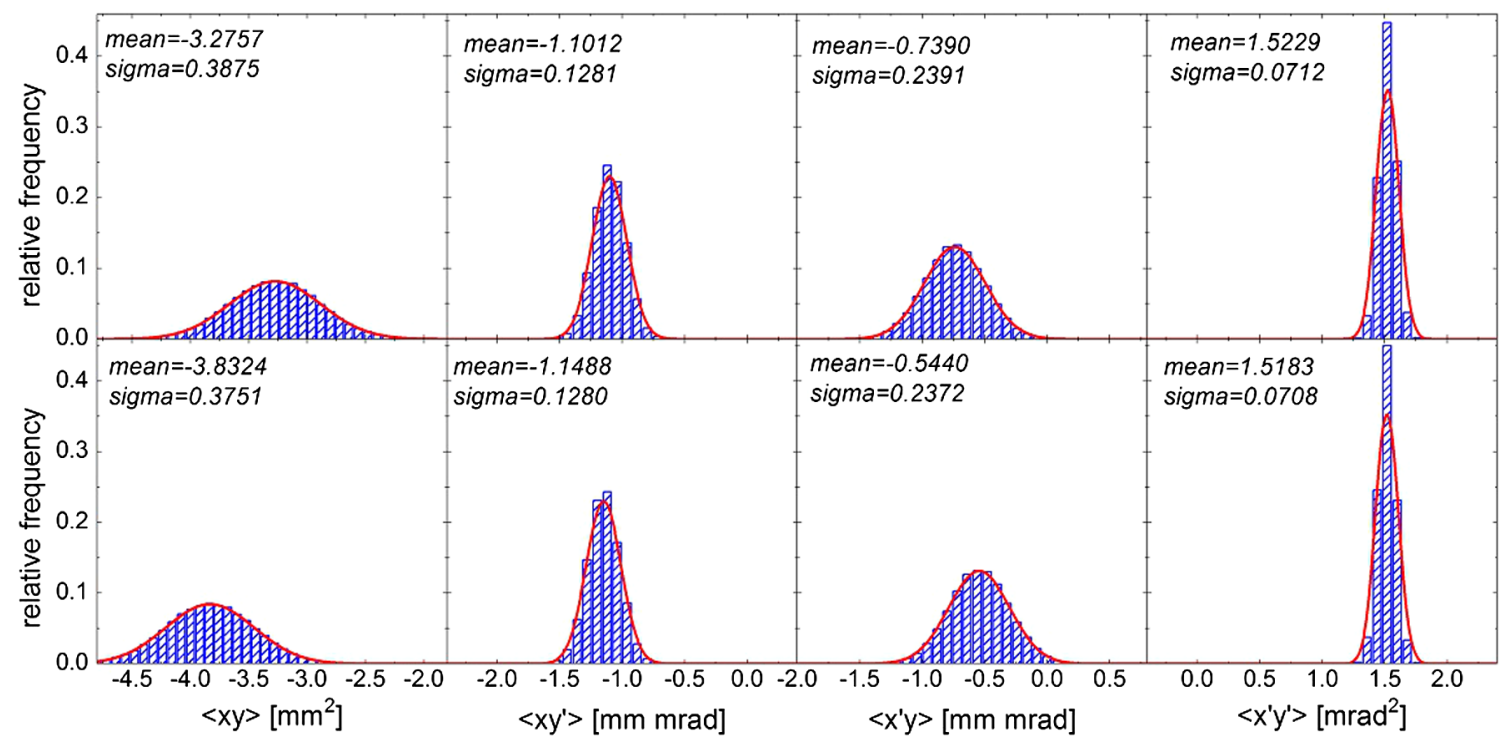

FIG. 8. Relative frequencies of the coupled second moments with skews on, the upper and lower pictures indicate the results with errors applying four and six measurements. Blue columns indicate the coupled second moments with errors and red solid lines indicate fits using Gaussian functions. The modified coupled second moments are evaluated as $\langle x y\rangle=-3.28 \pm 0.39 /-3.83 \pm 0.38 \mathrm{~mm}^{2},\left\langle x y^{\prime}\right\rangle=$ $-1.10 \pm 0.13 /-1.15 \pm 0.13 \mathrm{mmmrad},\left\langle x^{\prime} y\right\rangle=-0.74 \pm 0.24 /-0.54 \pm 0.24 \mathrm{mmmrad}$, and $\left\langle x^{\prime} y^{\prime}\right\rangle=-1.52 \pm 0.07 /-1.52 \pm 0.07 \mathrm{mrad}^{2}$. 


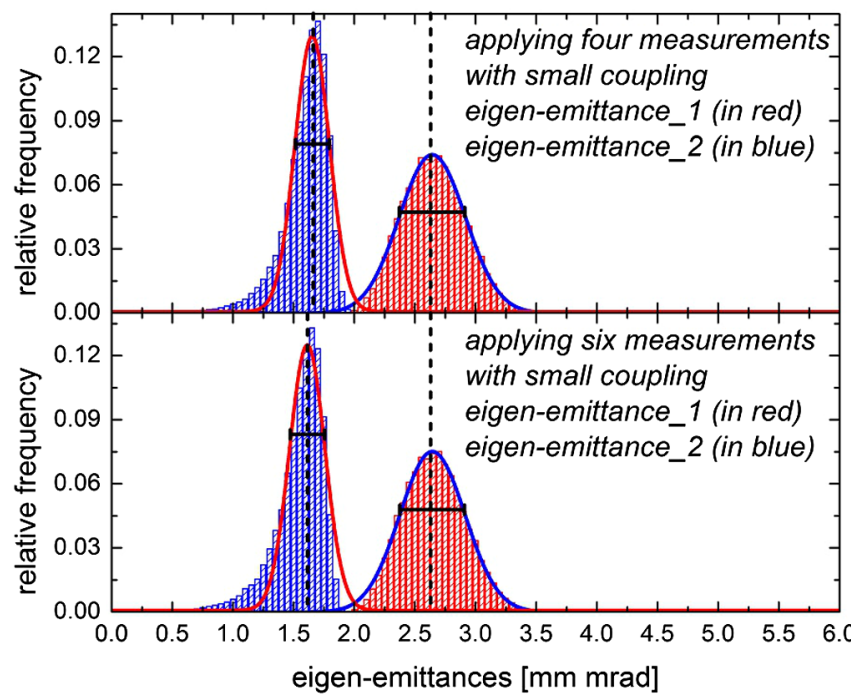

FIG. 9. Relative frequencies of the eigen emittances with skews off. The upper and lower pictures indicate the results applying four and six measurements, respectively. Red and blue columns indicate the eigen emittances and blue and red solid lines indicate fits using Gaussian functions. Black dashed lines indicate the measured eigen emittances without errors, and black solid lines indicate the rms error spans. Applying error analysis, the eigen emittances are $\varepsilon_{1}=2.64 \pm 0.27 / 2.64 \pm 0.27 \mathrm{~mm} \mathrm{mrad}$ and $\varepsilon_{2}=1.65 \pm 0.14 / 1.61 \pm 0.14 \mathrm{~mm}$ mrad.

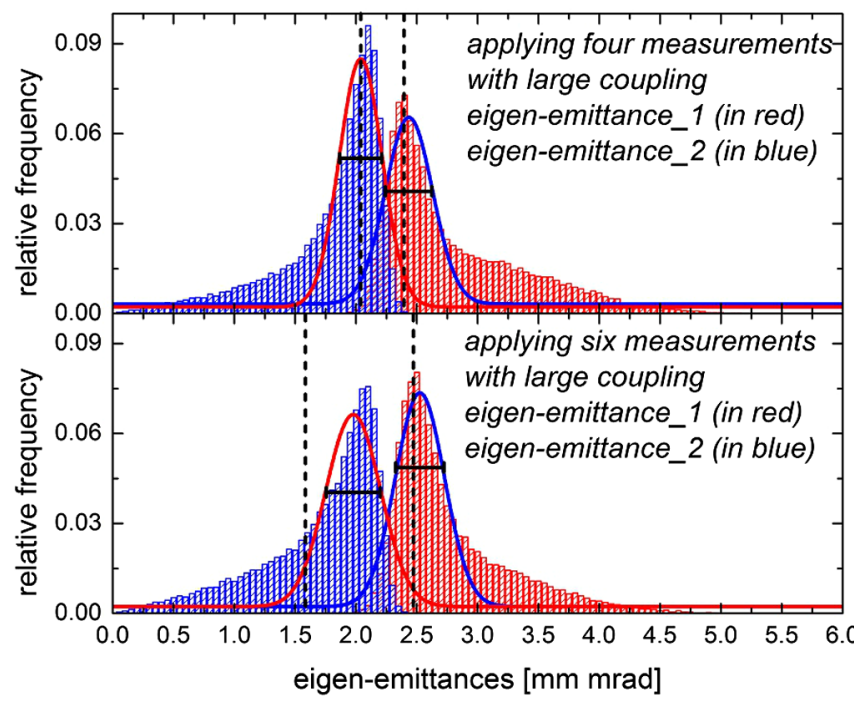

FIG. 10. Relative frequencies of the eigen emittances with skews on. The upper and lower pictures indicate the results applying four and six measurements, respectively. Red and blue columns indicate the eigen emittances and blue and red solid lines indicate fits using Gaussian functions. Black dashed lines indicate the measured eigen emittances without errors, and black solid lines indicate the rms error spans. Applying error analysis, the eigen-emittances are $\varepsilon_{1}=2.43 \pm 0.19 / 2.53 \pm 0.20 \mathrm{~mm} \mathrm{mrad}$ and $\varepsilon_{2}=2.04 \pm 0.17 / 1.98 \pm 0.22 \mathrm{~mm}$ mrad.

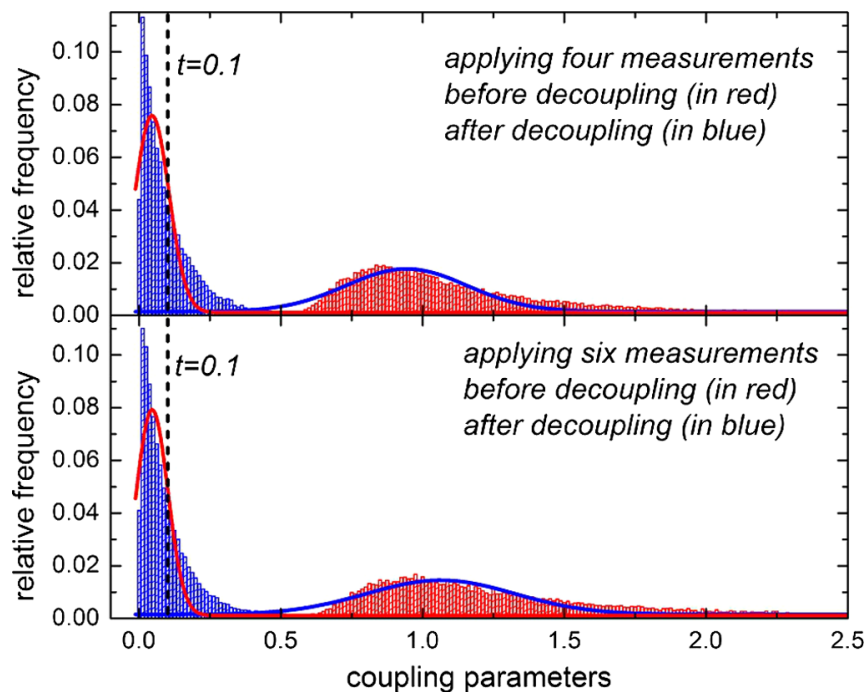

FIG. 11. Relative frequencies of the coupling parameters. The upper and lower pictures indicate the results applying Eq. (41) and Eq. (42). Red and blue columns indicate the values before and after decoupling and blue and red solid lines indicate fits using Gaussian functions. Applying the identical transfer matrix $R(4)$, the coupling parameters are reduced from $t=0.94 \pm 0.21 / 1.06 \pm 0.26$ to $t=0.05 \pm 0.06 / 0.05 \pm 0.05$.

$$
\begin{aligned}
\tilde{C}^{d}(4) & =R(4) \tilde{C}(4) R(4)^{T} \\
& =\left[\begin{array}{cccc}
9.80 & 0.00 & -0.17 & 0.04 \\
0.00 & 0.43 & -0.04 & -0.01 \\
-0.17 & -0.04 & 6.62 & 0.00 \\
0.04 & -0.01 & 0.00 & 0.86
\end{array}\right] .
\end{aligned}
$$

In the following this transfer matrix $R(4)$ is applied to the beam matrix $\tilde{C}(6)$

$$
\begin{aligned}
\tilde{C}^{d}(6) & =R(4) \tilde{C}(6) R(4)^{T} \\
& =\left[\begin{array}{cccc}
9.62 & 0.15 & -0.03 & -0.03 \\
0.15 & 0.27 & -0.09 & 0.08 \\
-0.03 & -0.09 & 6.78 & 0.00 \\
-0.03 & 0.08 & 0.00 & 0.89
\end{array}\right] .
\end{aligned}
$$

The coupling parameter $t$ of beam matrices $\tilde{C}^{d}(4)$ and $\tilde{C}^{d}(6)$ are evaluated to be $6.3 \times 10^{-4}$ and $1.4 \times 10^{-2}$, i.e. the beam is practically decoupled. The decoupling transfer matrix $R(4)$, constructed from four measurements will decouple the case from six measurements. The decoupling beam line is sensitive to the rms ellipses in the different projections. It is not sensitive to their eigen emittances and coupling parameters.

If the measurement errors are considered, the coupling parameters before and after the decoupling beam line using identical transfer matrix $R(4)$ are evaluated and shown in Fig. 11. 
After decoupling the beam line, most of the coupling parameters of decoupled beam matrices $\tilde{C}^{d}(4)$ and $\tilde{C}^{d}(6)$ are smaller than $t=0.1$, i.e. the beams are practical decoupled. Accordingly, even for beams being considerably coupled, just four measurements are required to determine the four-dimensional beam parameters with sufficient precision to allow for elimination of all interplane correlations by an appropriate beam line.

\section{CONCLUSION}

A new method using an rotatable slit and grid emittance measurement device called ROSE has been developed and commissioned to measure the four-dimensional second order beam matrix. It will allow precise and mobile four-dimensional emittance measurements. This unique setup works with high reliability. During ROSE commissioning it was found that three of the parameters extracted from the four-dimensional beam matrix (eigen emittance $\varepsilon_{1,2}$ and the $t$ parameter) are quite sensitive even to very small errors in the measurements, especially for notably correlated beams $t>1$. Despite careful choice of the optics reducing this sensitivity, fluctuations in $\varepsilon_{1,2}$ and $t$ were observed for a beam with considerable correlation. This observation confirms results from earlier single-plane emittance measurements, that featured large errors in case the beam was correlated. However, ROSE provides as major deliverable the optics to fully decouple a correlated beam. This optics is quite insensitive to the exact value of $\varepsilon_{1,2}$ and $t$ as it just depends on the second moments. The latter can be measured with sufficient precision. ROSE therefore provides the input for advanced coupled beam dynamics methods as the four-dimensional beam envelope model [32-36], for instance.

\section{ACKNOWLEDGMENTS}

C. X. expresses his sincere thanks to Peter Forck at GSI for fruitful discussions.

\section{APPENDIX A: MEASURED PROJECTED RMS EMITTANCES AND TWISS PARAMETERS WITH ERRORS}

The corresponding projected rms emittances and Twiss parameters with errors (mean $\pm \sigma$ ) at the entrance to the ROSE beam line are listed in Table IV.

\section{APPENDIX B: RMS EMMITTANCE ERROR}

Equation (2) states

$$
\varepsilon=\sqrt{\langle x x\rangle\left\langle x^{\prime} x^{\prime}\right\rangle-\left\langle x x^{\prime}\right\rangle^{2}}=\sqrt{\frac{\langle x x\rangle\left\langle x^{\prime} x^{\prime}\right\rangle}{1+\alpha^{2}}}
$$

and since second moments $\langle x x\rangle,\left\langle x x^{\prime}\right\rangle$, and $\left\langle x^{\prime} x^{\prime}\right\rangle$ are independent, the total error of the measured emittance $\delta \varepsilon$ is written as

$$
\delta \varepsilon=\sqrt{\left(\frac{\partial \varepsilon}{\partial\langle x x\rangle} \delta\langle x x\rangle\right)^{2}+\left(\frac{\partial \varepsilon}{\partial\left\langle x^{\prime} x^{\prime}\right\rangle} \delta\left\langle x^{\prime} x^{\prime}\right\rangle\right)^{2}+\left(\frac{\partial \varepsilon}{\partial\left\langle x x^{\prime}\right\rangle} \delta\left\langle x x^{\prime}\right\rangle\right)^{2}}
$$

where $\delta\langle x x\rangle, \delta\left\langle x x^{\prime}\right\rangle$, and $\delta\left\langle x^{\prime} x^{\prime}\right\rangle$ are the errors of the measured second moments. Accordingly,

TABLE IV. Projected rms emittances and Twiss parameters at the entrance of the ROSE beam line with errors. For $x-x^{\prime}, y-y^{\prime}$, $x-y^{\prime}$, and $y-x^{\prime}$ phase spaces are in units of mm mrad, 1 , and $\mathrm{m}$. For $x-y$ phase space is in units of $\mathrm{mm} \mathrm{mm}, 1$, and 1 . For $x^{\prime}-y^{\prime}$ phase

\begin{tabular}{|c|c|c|c|c|c|c|c|c|}
\hline Parameters & Skew triplet & Measurements & $x-x^{\prime}$ & $y-y^{\prime}$ & $x-y$ & $x^{\prime}-y^{\prime}$ & $x-y^{\prime}$ & $x^{\prime}-y$ \\
\hline$\varepsilon_{\mathrm{rms}}$ & & & $1.93 \pm 0.05$ & $2.46 \pm 0.37$ & $4.90 \pm 0.10$ & $1.59 \pm 0.25$ & $2.40 \pm 0.36$ & $3.25 \pm 0.07$ \\
\hline$\alpha_{\mathrm{rms}}$ & Off & Four & $1.32 \pm 0.04$ & $-0.01 \pm 0.05$ & $0.03 \pm 0.03$ & $0.14 \pm 0.04$ & $0.07 \pm 0.03$ & $0.09 \pm 0.05$ \\
\hline$\beta_{\mathrm{rms}}$ & & & $2.48 \pm 0.04$ & $2.01 \pm 0.30$ & $0.98 \pm 0.02$ & $1.32 \pm 0.18$ & $1.96 \pm 0.27$ & $1.55 \pm 0.03$ \\
\hline$\varepsilon_{\mathrm{rms}}$ & & & $1.93 \pm 0.05$ & $2.46 \pm 0.37$ & $4.90 \pm 0.10$ & $1.60 \pm 0.25$ & $2.37 \pm 0.37$ & $3.25 \pm 0.07$ \\
\hline$\alpha_{\mathrm{rms}}$ & Off & Six & $1.32 \pm 0.04$ & $-0.01 \pm 0.05$ & $0.02 \pm 0.03$ & $0.08 \pm 0.04$ & $0.18 \pm 0.04$ & $0.08 \pm 0.05$ \\
\hline$\beta_{\mathrm{rms}}$ & & & $2.48 \pm 0.04$ & $2.01 \pm 0.28$ & $0.98 \pm 0.02$ & $1.31 \pm 0.18$ & $1.99 \pm 0.28$ & $1.54 \pm 0.03$ \\
\hline$\varepsilon_{\mathrm{rms}}$ & & & $3.15 \pm 0.08$ & $3.66 \pm 0.65$ & $9.55 \pm 0.84$ & $1.99 \pm 0.04$ & $3.86 \pm 0.07$ & $6.28 \pm 0.50$ \\
\hline$\alpha_{\mathrm{rms}}$ & On & Four & $1.38 \pm 0.03$ & $0.80 \pm 0.20$ & $0.34 \pm 0.05$ & $-0.77 \pm 0.03$ & $0.28 \pm 0.03$ & $0.18 \pm 0.04$ \\
\hline$\beta_{\mathrm{rms}}$ & & & $2.72 \pm 0.02$ & $3.27 \pm 0.19$ & $0.89 \pm 0.08$ & $1.69 \pm 0.05$ & $2.22 \pm 0.04$ & $1.90 \pm 0.15$ \\
\hline$\varepsilon_{\mathrm{rms}}$ & & & $3.15 \pm 0.08$ & $3.76 \pm 0.57$ & $9.49 \pm 0.78$ & $1.99 \pm 0.04$ & $3.85 \pm 0.07$ & $6.38 \pm 0.46$ \\
\hline$\alpha_{\mathrm{rms}}$ & On & Six & $1.38 \pm 0.03$ & $0.77 \pm 0.17$ & $0.40 \pm 0.05$ & $-0.77 \pm 0.03$ & $0.29 \pm 0.03$ & $0.08 \pm 0.04$ \\
\hline$\beta_{\mathrm{rms}}$ & & & $2.72 \pm 0.02$ & $3.25 \pm 0.19$ & $0.90 \pm 0.08$ & $1.69 \pm 0.05$ & $2.23 \pm 0.04$ & $1.92 \pm 0.14$ \\
\hline
\end{tabular}
space is in units of mrad mrad, 1 , and 1 . 
$\frac{\delta \varepsilon}{\varepsilon}=\sqrt{\frac{1+\alpha^{2}}{\langle x x\rangle\left\langle x^{\prime} x^{\prime}\right\rangle} \frac{\sqrt{\left(\frac{1+\alpha^{2}}{\beta} \varepsilon \delta\langle x x\rangle\right)^{2}+\left(\beta \varepsilon \delta\left\langle x^{\prime} x^{\prime}\right\rangle\right)^{2}+4\left(\alpha \varepsilon \delta\left\langle x x^{\prime}\right\rangle\right)^{2}}}{2 \varepsilon}}=\frac{\sqrt{\left(\frac{1+\alpha^{2}}{\beta} \delta\langle x x\rangle\right)^{2}+\left(\beta \delta\left\langle x^{\prime} x^{\prime}\right\rangle\right)^{2}+4\left(\alpha \delta\left\langle x x^{\prime}\right\rangle\right)^{2}}}{2 \varepsilon}$.

Large $\alpha$ will cause large $\delta \varepsilon / \varepsilon$ for emittance measurements. In turn $\delta \varepsilon / \varepsilon$ is minimized for $\alpha=0$, i.e. for an uncoupled beam.

[1] C. Lejeune and J. Aubert, Emittance and Brightness: Definitions and Measurements in Applied Charged Particle Optics, Part A, edited by A. Septier (Academic Press, New York, 1980), pp. 159-259.

[2] O. R. Sander, Transverse Emittance: Its Definition, Applications, and Measurement, in Proceedings of the Accelerator Instrumentation Workshop, edited by E. R. Beadle and V. J. Castillo (AIP Conference Proceedings, New York, 1991), Vol. 212, pp. 127-155.

[3] S. Rimjaem et al., Optimizations of transverse projected emittance at the photo-injector test facility at DESY, location Zeuthen, Nucl. Instrum. Methods Phys. Res., Sect. A 671, 62 (2012).

[4] L. Groening, M. Maier, C. Xiao, L. Dahl, P. Gerhard, O. K. Kester, S. Mickat, H. Vormann, and M. Vossberg, Experimental Proof of Adjustable Single-Knob Ion Beam Emittance Partitioning, Phys. Rev. Lett. 113, 264802 (2014).

[5] V. Mironov, S. Bogomolov, A. Bondarchenko, A. Efremov, and V. Loginov, Numerical model of electron cyclotron resonance ion source, Phys. Rev. ST Accel. Beams 18, 123401 (2015).

[6] P. Spädtke, K. Tinschert, R. Lang, J. Mäder, J. R. bach, J. W. Stetson, and L. Celona, Prospects of ion beam extraction and transport simulations, Rev. Sci. Instrum. 79, 02B716 (2008).

[7] P. Spädtke, R. Lang, J. Mäder, J. R. Bach, and K. Tinschert, Low Energy Beam Transport for Ion Beams created by an ECRIS, in Proceedings of 18th International Workshop on ECR Ion Sources, Chicago, Illinois, 2008, edited by R. C. Pardo and R. C. Vondrasek (Argonne National Lab, Chicago, 2009).

[8] L. Groening, Concept for controlled transverse emittance transfer within a linac ion beam, Phys. Rev. ST Accel. Beams 14, 064201 (2011).

[9] C. Xiao, L. Groening, and O. K. Kester, Collimation and decoupling of ECR source beams for brilliance optimization, Nucl. Instrum. Methods Phys. Res., Sect. A 738, 167 (2014).

[10] P. Forck, Lecture notes on beam diagnostics, Joint University Accelerator School (JUAS), 2011, http:// www-bd.gsi.de/conf/juas/juas_script.pdf.

[11] P. Li, J. X. Wu, Y. J. Yuan, Y. Q. Yang, and S. L. Yang, The Design of Transverse Emittance Measurement at HIRFLCSR, in Proceedings of 19th International Conference on Cyclotrons and Their Applications, Lanzhou, China, 2010.

[12] L. Catani, E. Chiadroni, A. Cianchi, S. Tazzari, and M. Boscolo, Design and characterization of a movable emittance meter for low-energy electron beams, Rev. Sci. Instrum. 77, 093301 (2006).
[13] S. Kondrashev, A. Barcikowski, B. Mustapha, P. N. Ostroumov, and N. Vinogradov, Development of a pepper pot emittance probe and its application for ECR ion beam studies, Nucl. Instrum. Methods Phys. Res., Sect. A 606, 296 (2009).

[14] H. R. Kremers, J. P. M. Beijers, and S. Brandenburg, A versatile emittance meter and profile monitor, in Proceedings of the 8th European Workshop on Beam Diagnostics and Instrumentation for Particle Accelerator, Venice, Italy, 2007, edited by I. Andrian and V. R. W. Schaa (Elettra, Trieste, 2008), pp. 195-197.

[15] T. Nagatomo, V. Tzoganis, M. Kase, O. Kamigaito, and T. Nakagawa, Development of a pepper-pot emittance meter for diagnostics of low-energy multiply charged heavy ion beams extracted from an ECR ion source, Rev. Sci. Instrum. 87, 02B920 (2016).

[16] P. Forck (private communication).

[17] C. Thomas, N. Delerue, and R. Bartolini, Single shot $3 \mathrm{GeV}$ electron transverse emittance with a pepper-pot, Nucl. Instrum. Methods Phys. Res., Sect. A 729, 554 (2013).

[18] J. Rees and L. Rivkin, Stanford Linear Accelerator Center Report No. SLAC-pub-3305, 1984.

[19] M. D. Woodley and P. E. Emma, Measurement and Correction of corss-plane coupling in transport line, in Proceedings of the 20th International Linear Accelerator Conference, Monterey, CA, 2000 (SLAC, Menlo Park, CA, 2000), p. 196.

[20] E. Prat and M. Aiba, Four-dimensional transverse beam matrix measurement using the multiple-quadrupole scan technique, Phys. Rev. ST Accel. Beams 17, 052801 (2014).

[21] J. Ögren, R. Ruber, V. Ziemann, and W. Farabolini, Measuring the full transverse beam matrix using a single octupole, Phys. Rev. ST Accel. Beams 18, 072801 (2015).

[22] F. Löhl, Diploma thesis, University of Hamburg, 2005.

[23] K. M. Hock and A. Wolski, Tomographic reconstruction of the full 4D transverse phase space, Nucl. Instrum. Methods Phys. Res., Sect. A 726, 8 (2013).

[24] K. M. Hock, M. G. Ibison, D. J. Holder, A. Wolski, and B. D. Muratori, Beam tomography in transverse normalised phase space, Nucl. Instrum. Methods Phys. Res., Sect. A 642, 36 (2011).

[25] C. Xiao, L. Groening, P. Gerhard, M. Maier, S. Mickat, and $\mathrm{H}$. Vormann, Measurement of the transverse fourdimensional beam rms-emittance of an intense uranium beam at $11.4 \mathrm{MeV} / \mathrm{u}$, Nucl. Instrum. Methods Phys. Res., Sect. A 820, 14 (2016).

[26] K. R. Crandall and D. P. Rusthoi, Los Alamos National Laboratory Report No. LA-UR-97-886, 1997. 
[27] A. J. Dragt, General moment invariants for linear Hamiltonian systems, Phys. Rev. A 45, 2572 (1992).

[28] C. Xiao, O. K. Kester, L. Groening, H. Leibrock, M. Maier, and M. Rottländer, Single-knob beam line for transverse emittance partitioning, Phys. Rev. ST Accel. Beams 16, 044201 (2013).

[29] "Drehmodul für eine Beschleunigeranlage" Patent DRN 2015102213505700DE, Deutsches Patent-und Markenamt. A copy can be obtained from the author.

[30] W. Barth et al., Advanced superconducting CW heavy ion lianc R\&D, in Proceedings of the 4th International Particle Accelerator Conference, IPAC-2013, Shanghai, China, 2013 (JACoW, Shanghai, China, 2013).

[31] L. V. Foster, Rank and null space calculations using matrix decomposition without column interchanges, Linear Algebra Appl. 74, 47 (1986).

[32] H. Qin, M. Chung, and R. C. Davidson, Generalized Kapchinskij-Vladimirskij Distribution and Envelope
Equation for High-Intensity Beams in a Coupled Transverse Focusing Lattice, Phys. Rev. Lett. 103, 224802 (2009).

[33] M. Chung, H. Qin, E. P. Gilson, and R. C. Davidson, Analysis of continuously rotating quadrupole focusing channels using generalized Courant-Snyder theory, Phys. Plasmas 20, 083121 (2013).

[34] H. Qin, R. C. Davidson, M. Chung, and J. W. Burby, Generalized Courant-Snyder Theory for Charged-Particle Dynamics in General Focusing Lattices, Phys. Rev. Lett. 111, 104801 (2013).

[35] H. Qin, R. C. Davidson, J. W. Burby, and M. Chung, Analytical methods for describing charged particle dynamics in general focusing lattices using generalized CourantSnyder theory, Phys. Rev. ST Accel. Beams 17, 044001 (2014).

[36] M. Chung, H. Qin, L. Groening, R. C. Davidson, and C. Xiao, Beam envelope calculations in general linear coupled lattices, Phys. Plasmas 22, 013109 (2015). 